\title{
Normative data for the Brazilian population in the Boston Diagnostic Aphasia Examination: influence of schooling
}

M. Radanovic ${ }^{1}$, L.L. Mansur ${ }^{2}$ and M. Scaff ${ }^{1}$

\author{
Departamentos de ${ }^{1}$ Neurologia, and ${ }^{2}$ Fisioterapia, Fonoaudiologia e \\ Terapia Ocupacional, Faculdade de Medicina, Universidade de \\ São Paulo, São Paulo, SP, Brasil
}

\section{Correspondence}

M. Radanovic

Rua Cristiano Viana, 163/92

05411-000 São Paulo, SP

Brasil

Fax: +55-11-3088-9708

E-mail: radano@tecway.com.br

Publication supported by FAPESP.

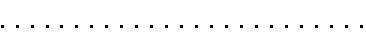

Received February 26, 2004

Accepted July 21, 2004

\begin{abstract}
In Neurolinguistics, the use of diagnostic tests developed in other countries can create difficulties in the interpretation of results due to cultural, demographic and linguistic differences. In a country such as Brazil, with great social contrasts, schooling exerts a powerful influence on the abilities of normal individuals. The objective of the present study was to identify the influence of schooling on the performance of normal Brazilian individuals in the Boston Diagnostic Aphasia Examination (BDAE), in order to obtain reference values for the Brazilian population. We studied 107 normal subjects ranging in age from 15 to 84 years (mean $\pm \mathrm{SD}=47.2 \pm 17.6$ years), with educational level ranging from 1 to 24 years ( $9.9 \pm 4.8$ years). Subjects were compared for scores obtained in the 28 subtests of the BDAE after being divided into groups according to age ( 15 to $30, \mathrm{~N}=24,31$ to $50, \mathrm{~N}=33$ and 51 years or more, $\mathrm{N}=50$ ) and education ( 1 to $4, \mathrm{~N}$ $=26,5$ to $8, N=17$ and 9 years or more, $N=61$ ). Subjects with 4 years or less of education performed poorer in Word Discrimination, Visual Confrontation Naming, Reading of Sentences and Paragraphs, and Primer-Level Dictation $(\mathrm{P}<0.05)$. When breakdown by schooling was 8 years or less, subjects performed poorer in all subtests $(\mathrm{P}<$ $0.05)$, except Responsive Naming, Word Recognition and WordPicture Matching. The elderly performed poorer $(\mathrm{P}<0.05)$ in Complex Ideational Material, Visual Confrontation Naming, Comprehension of Oral Spelling, Written Confrontation Naming, and Sentences to Dictation. We present the reference values for the cut-off scores according to educational level.
\end{abstract}

\section{Introduction}

Language abilities are strongly influenced by sociocultural characteristics. For this reason, we have been trying to determine the
Key words

- Boston Diagnostic Aphasia

Examination

- Schooling

- Brazilian population performance of the Brazilian population in tests of wide application in the diagnosis of aphasia, such as the Boston Diagnostic Aphasia Examination (BDAE) (1). In a previous study, we evaluated a sample of 60 normal 
subjects and tried to determine which demographic variables could influence their performance in the BDAE, as well as to obtain reference values for our population (2).

In the original validation of the BDAE for the American population there was no specific concern about adjusting the scores for subgroups of different sociocultural levels. Yet Pineda et al. (3), in a study conducted in Colombia, observed the importance of this correction. In Brazil, a country in which social differences are also extreme, it is expected that educational level may represent a powerful influence on the performance of subjects in neuropsychological tests in general $(4,5)$. However, in the study cited above (2) we were unable to find unequivocal differences. Thus, we realized that it was necessary to increase the sample size to include different educational levels.

The formation of the less educated group was not a straightforward task, because it is becoming increasingly harder to find participants less than 30 years old who report that they have not attended school, thanks to the implantation of policies to eradicate illiteracy in Brazil since the mid 1980's $(6,7)$. However, these efforts do not guarantee that these individuals will score well in tasks that involve formal knowledge, and often these subjects are in the functional illiteracy range (8).

On the other hand, the modern world provides mass communication media that are accessible to all population segments, leading to high exposure to the literate world, thus minimizing in some ways the deficiency of knowledge acquisition at school and attenuating these differences to a certain degree.

The purpose of the present study was to determine in greater detail the influence of schooling on the performance of Brazilian subjects on the BDAE, and to obtain more precise reference values for our population derived from a sample larger than the one of the previous study (2).

\section{Participants and Methods}

The Portuguese version of the BDAE (1) was applied to 107 Brazilian volunteers (64 women and $43 \mathrm{men}$ ), who were healthy, independent regarding daily life activities, without neurological or psychiatric antecedents, and who were all native Portuguese speakers living in São Paulo for more than 10 years. Subjects were recruited among the relatives of speech therapy students, hospital employees and outpatients' relatives or caregivers, with care taken to form groups that could represent age and schooling influences on the performance of normal individuals. Functional and conversational fluency evaluations were not considered for this study.

Speech therapy students were trained in the administration of the BDAE and applied the test using a uniform procedure while a speech therapy professor reviewed the scores obtained. Data were analyzed using the SPSS $^{\circledR}$ version 10.0 and MedCalc ${ }^{\circledR}$ version 7.2.1.0 software. To evaluate the effect of formal education, the subjects were divided into two groups: up to 8 years of study $(\mathrm{N}=$ $43)$, and 9 years or more $(\mathrm{N}=61)$. The group with up to 8 years of education was then further divided into two subgroups: up to 4 years $(\mathrm{N}=26)$, and from 5 to 8 years $(\mathrm{N}=17)$ of schooling for further comparison. In order to evaluate the age effect, the subjects were divided into three groups: $\mathrm{A}, 15$ to 30 years $(\mathrm{N}=24) ; \mathrm{B}, 31$ to 50 years $(\mathrm{N}=33) ; \mathrm{C}, 51$ years or more $(\mathrm{N}=50)$.

The distribution of the data for all the BDAE scores was tested by the Kolmogorov-Smirnov test, which showed a non-normal distribution. Since normal distribution could not be obtained after attempts at conversion, analysis was done using the nonparametric Kruskal-Wallis and MannWhitney tests to compare the performance of the different age and educational level subgroups in the various subtests. Inter-group differences were evaluated with the addition 
of the Dunn's post-test to the previous analysis. The results for animal fluency (which had a normal distribution) were compared by ANOVA followed by the Tukey-Kramer post-test. A value of $\mathrm{P}<0.05$ was considered to be statistically significant for all analyses.

\section{Results}

The age of the normal subjects studied ranged from 15 to 84 years (mean $\pm \mathrm{SD}=$ $47.2 \pm 17.6$ years), and educational level, defined as time devoted to formal study,

Table 1. Performance of subjects on the Boston Diagnostic Aphasia Examination ( $N=107)$ and recommended cut-off scores as a function of years of schooling.

\begin{tabular}{|c|c|c|c|c|c|}
\hline Subtest & Mean (SD) & $\begin{array}{c}95 \% \\
\text { confidence } \\
\text { index }\end{array}$ & Range & $\begin{array}{l}\text { Cut-off } \\
\text { score } \\
\text { (1-8 years) }\end{array}$ & $\begin{array}{c}\text { Cut-off score } \\
\text { (9 years } \\
\text { or more) }\end{array}$ \\
\hline \multicolumn{6}{|l|}{ Oral Comprehension } \\
\hline Word Discrimination & $70.6(3.5)$ & 69.9 to 71.3 & 48 to 72 & 60 & 68 \\
\hline Body-Part Identification & $19.5(0.9)$ & 19.3 to 19.7 & 15.5 to 20 & 17 & 19 \\
\hline Commands & $14.7(1)$ & 14.5 to 14.9 & 10 to 15 & 12 & 14 \\
\hline Complex Ideational Material & $10.8(1.5)$ & 10.5 to 11 & 5 to 12 & 6 & 10 \\
\hline \multicolumn{6}{|l|}{ Oral Agility } \\
\hline Nonverbal Agility & $9.1(2.1)$ & 8.7 to 9.5 & 4 to 12 & 4 & 6 \\
\hline Verbal Agility & $12.6(1.7)$ & 12.2 to 12.9 & 7 to 14 & 8 & 10 \\
\hline Automatized Sequences & $7.8(0.4)$ & 7.8 to 8 & 6 to 8 & 7 & 7 \\
\hline Recitation & $1.9(0.4)$ & 1.8 to 2 & 0 to 2 & 1 & 2 \\
\hline \multicolumn{6}{|l|}{ Repetition } \\
\hline Words & $9.9(0.2)$ & 9.9 to 10 & 8 to 10 & 9 & 10 \\
\hline High-Probability Phrases & $7.8(0.7)$ & 7.6 to 7.9 & 6 to 8 & 6 & 7 \\
\hline Low-Probability Phrases & $7.7(0.7)$ & 7.5 to 7.8 & 6 to 8 & 6 & 7 \\
\hline \multicolumn{6}{|l|}{ Naming } \\
\hline Responsive & $26.8(0.6)$ & 26.7 to 26.9 & 24 to 27 & 26 & 26 \\
\hline Visual Confrontation & $111.2(5.9)$ & 110 to 112.3 & 74 to 114 & 92 & 106 \\
\hline Animal Fluency & $21.3(7.8)$ & 19.7 to 22.9 & 7 to 47 & 5 & 8 \\
\hline \multicolumn{6}{|l|}{ Oral Reading } \\
\hline Words & $29.6(1.4)$ & 29.3 to 29.9 & 19 to 30 & 25 & 29 \\
\hline Sentences & $9.8(0.9)$ & 9.6 to 10 & 3 to 10 & 7 & 9 \\
\hline \multicolumn{6}{|l|}{ Reading Comprehension } \\
\hline Symbol Discrimination & $9.9(0.4)$ & 9.8 to 9.9 & 8 to 10 & 9 & 10 \\
\hline Word Recognition & $7.9(0.4)$ & 7.8 to 8 & 5 to 8 & 7 & 7 \\
\hline Comprehension of Oral Spelling & $6.5(2.1)$ & 6.1 to 7 & 1 to 8 & 1 & 5 \\
\hline Word-Picture Matching & $9.7(1)$ & 9.5 to 10 & 1 to 10 & 6 & 9 \\
\hline Sentences and Paragraphs & $9.4(1)$ & 9.2 to 9.5 & 5 to 10 & 6 & 9 \\
\hline \multicolumn{6}{|l|}{ Writing } \\
\hline Writing Mechanics & $4.9(0.5)$ & 4.7 to 5 & 2 to 5 & 4 & 5 \\
\hline Serial Writing & $45 \quad(3.4)$ & 44.3 to 45.6 & 21 to 47 & 34 & 43 \\
\hline Primer-Level Dictation & $14.5(1.3)$ & 14.3 to 14.8 & 7 to 15 & 10 & 14 \\
\hline Spelling to Dictation & $9.3(1.5)$ & 9 to 9.6 & 2 to 10 & 4 & 9 \\
\hline Written Confrontation Naming & $9.6(1.3)$ & 9.4 to 9.9 & 6 to 10 & 5 & 9 \\
\hline Narrative Writing & $4.5(0.9)$ & 4.4 to 4.7 & 2 to 5 & 2 & 4 \\
\hline Sentences to Dictation & $11.6(1.5)$ & 11.3 to 11.9 & 6 to 12 & 6 & 11 \\
\hline
\end{tabular}


ranged from 1 to 24 years $(9.9 \pm 4.8$ years $)$

Table 1 shows the overall results for the 28 BDAE subtests. We present a suggested cut-off score for use in Brazil, calculated as the mean minus two SD and corrected for educational level. Table 2 shows the overall distribution of the results in percentiles.

Table 2. Performance of subjects on the Boston Diagnostic Aphasia Examination

\begin{tabular}{|c|c|c|c|c|c|c|c|c|}
\hline \multirow[t]{2}{*}{ Subtest } & \multicolumn{8}{|c|}{ Percentile } \\
\hline & 5 & 10 & 25 & 50 & 75 & 90 & 95 & 100 \\
\hline \multicolumn{9}{|l|}{ Oral Comprehension } \\
\hline Word Discrimination & 62 & 67 & 72 & & & & & \\
\hline Body-Part Identification & 17 & 18 & 19 & 20 & & & & \\
\hline Commands & 11 & 15 & & & & & & \\
\hline Complex Ideational Material & 8 & 9 & 10 & 11 & 12 & & & \\
\hline \multicolumn{9}{|l|}{ Oral Agility } \\
\hline Nonverbal Agility & 6 & & 7 & 9 & 11 & 12 & & \\
\hline Verbal Agility & 9 & 10 & 12 & 13 & 14 & & & \\
\hline Automatized Sequences & & & & & & & & \\
\hline Recitation & 1 & 2 & & & & & & \\
\hline \multicolumn{9}{|l|}{ Repetition } \\
\hline Words & 10 & & & & & & & \\
\hline High-Probability Phrases & 7 & & 8 & & & & & \\
\hline Low-Probability Phrases & 6 & 7 & 8 & & & & & \\
\hline \multicolumn{9}{|l|}{ Naming } \\
\hline Responsive & 26 & & 27 & & & & & \\
\hline Visual Confrontation & 98 & 105 & 111 & 114 & & & & \\
\hline Animal Fluency & 11 & 12 & 15 & 20 & 27 & 32 & 34 & 40 \\
\hline \multicolumn{9}{|l|}{ Oral Reading } \\
\hline Words & 27 & 29 & 30 & & & & & \\
\hline Sentences & 9 & 10 & & & & & & \\
\hline \multicolumn{9}{|l|}{ Reading Comprehension } \\
\hline Symbol Discrimination & 9 & & 10 & & & & & \\
\hline Word Recognition & 8 & & & & & & & \\
\hline Comprehension of Oral Spelling & 1 & 3 & 6 & 8 & & & & \\
\hline Word-Picture Matching & 9 & 10 & & & & & & \\
\hline Sentences and Paragraphs & 8 & 8 & 9 & 10 & & & & \\
\hline \multicolumn{9}{|l|}{ Writing } \\
\hline Writing Mechanics & 4 & & 5 & & & & & \\
\hline Serial Writing & 39 & 43 & 44 & 46 & 47 & & & \\
\hline Primer-Level Dictation & 13 & 14 & 15 & & & & & \\
\hline Spelling to Dictation & 6 & 8 & 9 & 10 & & & & \\
\hline Written Confrontation Naming & 8 & 9 & 10 & & & & & \\
\hline Narrative Writing & 3 & & 4 & 5 & & & & \\
\hline Sentences to Dictation & 9 & 11 & 12 & & & & & \\
\hline
\end{tabular}

Data are reported for 107 subjects in percentile.
Comparison of performance among the three age groups revealed a significant difference in the Complex Ideational Material, Visual Confrontation Naming, Comprehension of Oral Spelling, Written Confrontation Naming, and Sentences to Dictation subtests (Table 3). The mean educational level for the three groups was $12.5 \pm 2.3$ years for group A, $10.7 \pm 5.1$ years for group B, and $7.8 \pm 4.8$ years for group C. There was a significant difference in average educational level among these groups, as follows: group $\mathrm{A}$ vs $\mathrm{C}(\mathrm{P}<0.001)$ and group $\mathrm{B}$ vs $\mathrm{C}(\mathrm{P}=$ $0.019)$.

Educational level (1 to 8 vs 9 years or more) influenced performance in all subtests, except Responsive Naming, Word Recognition and Word-Picture Matching (Table 4). However, the comparison between the subgroups with 1 to 4 and 5 to 8 years revealed significant differences in Word Discrimination, Visual Confrontation Naming, Comprehension of Sentences and Paragraphs, and Primer-Level Dictation (Table 5).

\section{Discussion}

The content of a language test must reflect the basic linguistic knowledge of a particular social group. In fact, our results show that subjects with less than 8 years of education behave in a relatively homogeneous way. This becomes more evident as they are subdivided into subgroups of 1 to 4 and 5 to 8 years of education, when there are no differences in most of the subtests, except for Word Discrimination, Visual Confrontation Naming, Comprehension of Sentences and Paragraphs, and Primer-Level Dictation (Table 5). Indeed, Word Discrimination, Visual Confrontation Naming and Primer-Level Dictation are all tasks that demand a greater degree of semantic knowledge (especially because they involve geometric shapes and syntactically complex numbers), whereas the Comprehension of Sentences and Paragraphs task requires a reading ability, which im- 
proves with increasing years of formal education.

When breakdown by schooling was 8 years or less we observed differences in all subtests, except for Responsive Naming, Word Recognition and Word-Picture Matching (Table 4). The tasks for which this difference was more marked were Word Discrimination, Body Part Identification, Complex Ideational Material, Animal Fluency, Comprehension of Oral Spelling, Comprehension of Sentences and Paragraphs, Spelling to Dictation, Written Confrontation Naming, and Sentences to Dictation. The Word Discrimination subtest involves semantic categories whose knowledge is related to the experience frequently acquired at school, like geometric shapes and details of body parts. The comprehension and memorization of texts, necessary for the Complex Ideational Material and Comprehension of Sentences and Paragraphs tasks, demand the establishment of complex semantic relations in order to derive their meaning. The comprehension of texts is based on the analysis of propositions on a logical basis, necessary in the making of inferences, indicating the subject's ability to transpose his personal experience, both factual and concrete (9). It can be noticed that this difficulty was observed as much in the comprehension of oral texts as in the written texts (Comprehension of Sentences and Paragraphs), not surprisingly given that the oral texts in this task were in fact previously devised in the written modality, so that the oral presentation maintains the characteristics of the written language, such as the absence of redundancies, among others.

The influence of schooling on the generation of lexical items in a particular semantic category (animal) had already been studied in the Brazilian population (10). The knowledge of semantic categories, supplied by formal education, probably helps the management and organization of the semantic groups, leading to the generation of a greater number of lexical items by the more educated subjects.

The differences observed in the written subtests (Spelling to Dictation, Written Con-

Table 3. Performance of subjects on the Boston Diagnostic Aphasia Examination by age.

\begin{tabular}{|c|c|c|c|}
\hline \multirow[t]{2}{*}{ Subtest } & \multicolumn{3}{|c|}{ Age (years) } \\
\hline & A (15-30) & B $(31-50)$ & C (51 or more) \\
\hline \multicolumn{4}{|l|}{ Oral Comprehension } \\
\hline Word Discrimination & $71.6(1.4)$ & $70.7(3.2)$ & $70.1(4.2)$ \\
\hline Body-Part Identification & $19.5(0.9)$ & $19.5(0.9)$ & $19.4(0.9)$ \\
\hline Commands & $15(0)$ & $14.7(1.1)$ & $14.5(1.2)$ \\
\hline Complex Ideational Material* & $11.3(1.2)$ & $11.1(1.4)$ & $10.3(1.6)$ \\
\hline \multicolumn{4}{|l|}{ Oral Agility } \\
\hline Nonverbal Agility & $9.7(1.8)$ & $9.3(2.1)$ & $8.7(2.2)$ \\
\hline Verbal Agility & $13.1(1.2)$ & $12.8(1.6)$ & $12.1(1.9)$ \\
\hline Automatized Sequences & $7.9(0.4)$ & $7.8(0.3)$ & $7.7(0.5)$ \\
\hline Recitation & $1.9(0.2)$ & $1.8(0.4)$ & $1.9(0.2)$ \\
\hline \multicolumn{4}{|l|}{ Repetition } \\
\hline Words & $10(0)$ & $10(0)$ & $9.9(0.3)$ \\
\hline High-Probability Phrases & $7.9(0.4)$ & $7.8(0.4)$ & 7.7 (0.8) \\
\hline Low-Probability Phrases & $7.8(0.4)$ & $7.7(0.6)$ & $7.6(0.9)$ \\
\hline \multicolumn{4}{|l|}{ Naming } \\
\hline Responsive & $26.9(0.2)$ & $26.8(0.6)$ & $26.8(0.7)$ \\
\hline Visual Confrontation ${ }^{+}$ & $113.2(1.8)$ & $111.6(4.2)$ & $109.9(7.7)$ \\
\hline Animal Fluency & $23.5(9.7)$ & $22.6(6.7)$ & $19.4(7.3)$ \\
\hline \multicolumn{4}{|l|}{ Oral Reading } \\
\hline Words & $29.8(0.7)$ & $29.8(0.5)$ & $29.4(2)$ \\
\hline Sentences & $9.9(0.2)$ & $9.9(0.3)$ & $9.6(1.3)$ \\
\hline \multicolumn{4}{|l|}{ Reading Comprehension } \\
\hline Symbol Discrimination & $10(0)$ & $9.8(0.4)$ & $9.8(0.4)$ \\
\hline Word Recognition & $8(0.4)$ & $7.9(0.5)$ & $7.9(0.3)$ \\
\hline Comprehension of Oral Spelling** & $7.4(1.5)$ & $7(1.6)$ & $5.8(2.4)$ \\
\hline Word-Picture Matching & $9.8(0.6)$ & $9.7(1.6)$ & $9.8(0.8)$ \\
\hline Sentences and Paragraphs & $9.7(0.5)$ & $9.4(0.7)$ & $9.2(1.3)$ \\
\hline \multicolumn{4}{|l|}{ Writing } \\
\hline Writing Mechanics & $4.9(0.3)$ & $4.9(0.4)$ & $4.8(0.5)$ \\
\hline Serial Writing & $45.9(1.4)$ & $45.5(2)$ & $44.2(4.5)$ \\
\hline Primer-Level Dictation & $14.8(0.5)$ & $14.7(1)$ & $14.3(1.7)$ \\
\hline Spelling to Dictation & $9.8(0.4)$ & $9.6(0.9)$ & $8.9(2)$ \\
\hline Written Confrontation Naming ${ }^{++}$ & $10(0)$ & $9.8(0.7)$ & $9.4(1.7)$ \\
\hline Narrative Writing & $4.7(0.6)$ & $4.7(0.7)$ & $4.4(1)$ \\
\hline Sentences to Dictation\# & $12(0)$ & $11.9(0.4)$ & $11.2(2.1)$ \\
\hline
\end{tabular}

Data are reported as means (SD) for 107 subjects.

${ }^{*} \mathrm{P}<0.05$ for $\mathrm{A}$ vs $\mathrm{C}$ and $\mathrm{B}$ vs $\mathrm{C}$. ${ }^{+} \mathrm{P}<0.05$ for $\mathrm{A}$ vs $\mathrm{B}$ and $\mathrm{A}$ vs $\mathrm{C} .{ }^{* *} \mathrm{P}<0.05$ for $\mathrm{A}$ vs $\mathrm{C}$ and $\mathrm{B}$ vs $\mathrm{C}$; ${ }^{++} \mathrm{P}<0.05$ for $\mathrm{A}$ vs $\mathrm{C}$. $\# \mathrm{P}<0.05$ for $\mathrm{A}$ vs $\mathrm{C}$ and $\mathrm{B}$ vs $\mathrm{C}$ (Kruskal-Wallis with Dunn's post-test). 
frontation Naming and Sentences to Dictation) reflect the sensitivity of these tasks not only to the instrumental abilities related to the phoneme-grapheme conversion and spelling irregularities, but also to the ability of process-

Table 4. Performance of subjects on the Boston Diagnostic Aphasia Examination by educational level.

\begin{tabular}{|c|c|c|}
\hline \multirow[t]{2}{*}{ Subtest } & \multicolumn{2}{|c|}{ Years of schooling } \\
\hline & $1-8$ & 9 or more \\
\hline \multicolumn{3}{|l|}{ Oral Comprehension } \\
\hline Word Discrimination & $69.3(4.8)$ & $71.5(1.6)^{*}$ \\
\hline Body-Part Identification & $19.1(1.1)$ & $19.7(0.6)^{*}$ \\
\hline Commands & $14.4(1.4)$ & $14.9(0.7)^{*}$ \\
\hline Complex Ideational Material & $9.9(1.8)$ & $11.3(0.9)^{*}$ \\
\hline \multicolumn{3}{|l|}{ Oral Agility } \\
\hline Nonverbal Agility & $8.6(2.2)$ & $9.6(2)^{*}$ \\
\hline Verbal Agility & $11.9(2)$ & $13(1.3)^{*}$ \\
\hline Automatized Sequences & $7.7(0.6)$ & $7.9(0.3)^{*}$ \\
\hline Recitation & $1.8(0.4)$ & $1.9(0.2)^{*}$ \\
\hline \multicolumn{3}{|l|}{ Repetition } \\
\hline Words & $9.9(0.4)$ & $10(0)^{*}$ \\
\hline High-Probability Phrases & $7.6(0.9)$ & $7.9(0.3)^{*}$ \\
\hline Low-Probability Phrases & $7.5(1)$ & $7.8(0.4)^{*}$ \\
\hline \multicolumn{3}{|l|}{ Naming } \\
\hline Responsive & $26.8(0.6)$ & $26.9(0.5)$ \\
\hline Visual Confrontation & $109.2(8.4)$ & $112.4(3)^{*}$ \\
\hline Animal Fluency & $18.2(6.5)$ & $23.6(8)^{*}$ \\
\hline \multicolumn{3}{|l|}{ Oral Reading } \\
\hline Words & $29.3(2.2)$ & $29.9(0.5)^{*}$ \\
\hline Sentences & $9.5(1.4)$ & $9.9(0.2)^{*}$ \\
\hline \multicolumn{3}{|l|}{ Reading Comprehension } \\
\hline Symbol Discrimination & $9.7(0.5)$ & $9.9(0.2)^{*}$ \\
\hline Word Recognition & $7.9(0.5)$ & $8(0.3)$ \\
\hline Comprehension of Oral Spelling & $5.4(2.5)$ & $7.3(1.2)^{*}$ \\
\hline Word-Picture Matching & $9.5(1.6)$ & $9.9(0.4)$ \\
\hline Sentences and Paragraphs & $8.9(1.3)$ & $9.7(0.5)^{*}$ \\
\hline \multicolumn{3}{|l|}{ Writing } \\
\hline Writing Mechanics & $4.7(0.6)$ & $4.9(0.1)^{*}$ \\
\hline Serial Writing & $43.7(4.8)$ & $45.8(1.5)^{*}$ \\
\hline Primer-Level Dictation & $14.1(2)$ & $14.8(0.4)^{*}$ \\
\hline Spelling to Dictation & $8.5(2.2)$ & $9.9(0.3)^{*}$ \\
\hline Written Confrontation Naming & $9.2(1.9)$ & $9.9(0.2)^{*}$ \\
\hline Narrative Writing & $4.2(1.2)$ & $4.8(0.4)^{*}$ \\
\hline Sentences to Dictation & $11(2.3)$ & $11.9(0.2)^{*}$ \\
\hline
\end{tabular}

Data are reported as means (SD).

${ }^{*} \mathrm{P}<0.05$ compared 1 to 8 years of schooling (Mann-Whitney test). ing verbal material in short-term memory (11).

The results of the present study show that the continuum of changes effected by formal instruction goes beyond the first years of elementary school and reveal that the effects of its deprivation in language tasks can be felt throughout the "Fundamental Level" (the first 8 years of schooling in Brazil). In fact, 9 years of education are necessary for individuals to exhibit average scores that can almost equal the maximum possible score for each task - the expected result in a diagnostic test which has been designed to differentiate normal individuals from those neurologically impaired - where specificity is more important. It is interesting to note that the authors of the BDAE validated the test on a population whose education ranged from less than eight grade through college (12), and where the mean was within a fraction of a point of the maximum possible score in most subtests. The few exceptions are invariably found in the group of subjects over 60 years of age with fewer than 9 years of schooling (1).

Although there is wide propagation of knowledge by the communication media in modern life, thereby providing alternative ways for the acquisition of knowledge and for the cognitive functioning of less educated individuals, we observed that school attendance can still cause significant differences in the domain of metaknowledge requested by language tests such as the BDAE. In other words, this knowledge, although alternative, is not compensatory, as pointed out by Tfouni (13). On the other hand, formal education in school, by itself, does not guarantee the subject's engagement in the social practices that require the interpretation of complex texts or writing. Regarding this issue, it is well known that many individuals who attend school still exhibit poor linguistic skills and can be considered to be functional illiterates (when presenting reading competence compatible with 0 to 4 years of schooling) or to be marginally literate (com- 
patible with 5 to 8 years of schooling) $(8,14)$.

The analysis of performance by age subgroup showed that this variable had no influence on most subtests (Table 3). Although we detected differences in some tasks (Complex Ideational Material, Visual Confrontation Naming, Comprehension of Oral Spelling, Written Confrontation Naming, and Sentences to Dictation), the coexistence of statistically significant differences in educational level among these groups does not allow us to attribute these results to the variable age alone. All of these tasks were influenced by the educational level of the subjects.

Based on the differences in performance found in relation to schooling, we propose the use of two cut-off scores in our population, as shown in Table 1. Although this study was conducted in São Paulo, we believe that the reference values are valid for the Brazilian population as a whole, because the only lexical item that reflects a regional particularity for naming is "cactus", which in some regions of the country can also be named "mandacaru" or "palma". The remainder of the test contains items whose naming and meaning are the same for the whole country. It is worth emphasizing that the recognition of the limitations caused by the lack of education is essential in order to minimize the occurrence of falsepositive results for less educated individuals, leading to an overdiagnosis of language disturbances in normal subjects.

\section{Acknowledgments}

The authors would like to thank the undergraduate students of the Speech Pathology Course, Faculdade de Medicina, Universidade de São Paulo, for collecting the data.
Table 5. Performance of subjects on the Boston Diagnostic Aphasia Examination by educational level.

Subtest

Years of schooling

$1-4 \quad 5-8$

Oral Comprehension

Word Discrimination

Body-Part Identification

Commands

Complex Ideational Material

$68(5.7)$

$71.2(1.7)^{*}$

$18.9(1.3)$

$14.2(1.6)$

$19.5(0.6)$

$14.6(1)$

Oral Agility

Nonverbal Agility

$9.5(2)$

$10.6(1.3)$

Verbal Agility

$8.2(2.1)$

$9.1(2.3)$

Automatized Sequences

$11.7(1.6)$

$12.2(2.7)$

Recitation

$7.7(0.6)$

$7.6(0.5)$

$1.7(0.5)$

$1.9(0.2)$

Repetition

Words

High-Probability Phrases

$9.8(0.4)$

$7.5(1.2)$

Low-Probability Phrases

$7.3(1.2)$

$10(0)$

Naming

Responsive

$26.7(0.6)$

106.9 (9.9)

Visual Confrontation

18 (7)

Animal Fluency

$28.9(2.6)$

$9.3(1.8)$

$29.8(0.8)$

Words

Sentences

$9.6(0.6)$

$7.8(0.7)$

$5.1(2.7)$

$9.2(2)$

8.5 (1.5)

$7.7(0.4)$

$7.8(0.4)$

Reading Comprehension

Symbol Discrimination

Comprehension of Oral Spelling

Word-Picture Matching

Sentences and Paragraphs

$4.7(0.7)$

$43(5.5)$

$13.6(2.4)$

$8.2(2.4)$

$8.9(2.3)$

4 (1.3)

$10.8(2.7)$

$9.9(0.3)$

Serial Writing

Primer-Level Dictation

Written Confrontation Naming

Narrative Writing

Sentences to Dictation

$9.9(0.2)$

8 (0)

$5.7(2.3)$

$10(0)$

$9.5(0.6)^{*}$

Data are reported as means (SD)

${ }^{*} \mathrm{P}<0.05$ compared to $1-4$ years of schooling (Mann-Whitney test).

\section{References}

1. Goodglass H \& Kaplan E (1983). The Assessment of Aphasia and Related Disorders. 2nd edn. Lea \& Febiger, Philadelphia, PA, USA.

2. Radanovic M \& Mansur LL (2002). Performance of a Brazilian popu- lation sample in the Boston Diagnostic Aphasia Examination. A pilot study. Brazilian Journal of Medical and Biological Research, 35: 305317 
3. Pineda DA, Rosselli M, Ardila A, Mejia SE, Romero MG \& Pérez C (2000). The Boston Diagnostic Aphasia Examination - Spanish Version: Influence of demographic variables. Journal of the International Neuropsychological Society, 6: 802-814.

4. Bertolucci PHF, Brucki SMD, Campacci SR \& Juliano Y (1994). O Mini-Exame do Estado Mental em uma população geral. Impacto da escolaridade. Arquivos de Neuro-Psiquiatria, 52: 1-7.

5. Bertolucci PHF, Mathias SC, Brucki SMD, Carrilho PEM, Okamoto IH \& Nitrini R (1994). Proposta de padronização do Mini-Exame do Estado Mental (MEEM): estudo piloto cooperativo (FMUSP/EPM). Arquivos de Neuro-Psiquiatria, 52 (Suppl): P-225.

6. Di Pierro MC (2001). Decentralization and partnership: an assessment of the education of youngsters and adults in the Brazilian educational reform. Educação e Pesquisa, 27: 322-337.

7. Kleiman $A B$ (2001). Development programs and research: the contribution of literacy studies to the education of youngsters and adults. Educação e Pesquisa, 27: 268-281.

8. Moreira DA (2003). Analfabetismo Funcional: O Mal Nosso de Cada Dia. Thomson, São Paulo, SP, Brazil.
9. Kamhi AG \& Catts HW (1989). Language and reading convergences, divergences, and development. In: Kahmi AG \& Catts HW (Editors), Reading Disabilities - A Developmental Language Perspective. College-Hill Publication, Little, Brown and Company, Boston, MA, USA.

10. Brucki SM, Malheiros SM, Okamoto IH \& Bertolucci P (1997). Normative data on the verbal fluency test in the animal category in our milieu. Arquivos de Neuro-Psiquiatria, 55: 56-61.

11. Kolinsky R (1996). Conséquences cognitives de l'illetrisme. In: Carbonnell S, Gillet PM-D, Martory M-D \& Valdois S (Editors), Approche Cognitive des Troubles de la Lecture et de l'Écriture Chez l'Enfant et I'Adulte. Collection Neuropsychologie, Solal, Marseille, France.

12. Borod J, Goodglass H \& Kaplan E (1980). Normative data on the Boston Diagnostic Aphasia Examination, parietal lobe battery and Boston Naming Test. Journal of Clinical Neuropsychology, 2: 209216.

13. Tfouni LV (1988). Adultos Não Alfabetizados: O Avesso do Avesso. Pontes, Campinas, SP, Brazil.

14. Moreira DA (2003). Analfabetismo Funcional: O Mal Nosso de Cada Dia. Thomson, São Paulo, SP, Brazil. 